paradoxically harmful in IBD. However, disruption of the alternative pathway by deleting NFKB2 protected murine colon from developing inflammation and this was associated with reduced expression of TNF- $\alpha$ and IL-14. Pharmacological inhibition of the NFKB2 signalling pathway may therefore be a promising novel therapeutic strategy for IBD.

Competing interests None declared.

\section{PMO-225 USE OF INFLIXIMAB FOR ACUTE SEVERE ULCERATIVE COLITIS IN A DISTRICT GENERAL HOSPITAL}

doi:10.1136/gutjnl-2012-302514b.225

A Alisa,* S Musa, S Zar, A Mahmood. Gastroenterology, St Helier Hospital, London, UK

Introduction The prevalence of Ulcerative colitis (UC) in the UK is $243 / 100000$ and carries a high lifetime risk of surgery $(20 \%-30 \%)$. Acute severe UC (ASUC) is potentially a life threatening condition. Traditionally urgent medical treatment includes intravenous steroids followed by IV Ciclosporin in non-responders. In 2010, NICE recommended the use of Infliximab in ASUC in patients in whom ciclosporin is contraindicated or clinically inappropriate. Randomised control trials suggest colectomy is required in 30\% of Infliximab recipients at 3 months and $50 \%$ at 3 years. ${ }^{12}$ This retrospective study reports our experience at a busy district general hospital in South West London.

Methods The Biologic database at St. Helier's Hospital was interrogated to identify all cases of ASUC that gained funding for. Infliximab data collection was from January 2009 to June 2011. All patients had a UC disease activity index score (UCDAI) $>10$. Disease site was classified by the Montreal classification. Follow-up ranged from 6 to 36 months post treatment.

Results 20 patients had funding approved. 17/20 (85\%) patients received Infliximab, 3/20 (15\%) clinically settled pre-administration. There were $9(53 \%)$ males and 8 (47\%) females, median age 47 (range 19-57). 9 (53\%) patients had left sided disease and $8 / 17$ (47\%) had extensive colitis. Median UCDAI was 11 (range 10-13). The median disease duration 4 years (range $0-15$ years). During the acute presentation (pre-Infliximab), 11/17 (65\%) patients received steroids, $11 / 17$ (65\%) were already on azathioprine, 15/17 (88\%) 5-ASA, 3/17 (18\%) 6-MP and 1/17 (6\%) Tacrolimus. Following steroid therapy 4/ $17(24 \%)$ had received Ciclosporin. Urgent colectomy was required in $2 / 17$ (12\%) patients. A further 6/17 (35\%) underwent elective colectomy post induction, median 5 months (range $2-12$ months). Complications were experienced in 3/17 (18\%) and included 1 lichen planus, 1 Raynauds, 1 arthralgia. There was no mortality reported. Conclusion Following Infliximab therapy urgent colectomy was avoided in the majority ( $82 \%$ ) of patients. Our elective colectomy rate was not to dissimilar to those reported by main randomised control trials. Infliximab was well tolerated and provided rescue therapy prior to latent elective surgery.

Competing interests None declared.

\section{REFERENCES}

1. Jarnerot G, et al. Gastroenterology 2005;128:1805-11.

2. Gustavsson A, et al. Aliment Pharmacol Ther 2010;32:984-9.

\section{PM0-226 DIFFERING PHENOTYPE IN ELDERLY IBD; SHOULD MONTREAL INCLUDE AN A4 CATEGORY}

doi:10.1136/gutjnl-2012-302514b.226

A A McNicol, ${ }^{*}$ N Kennedy, J Satsangi, I D Arnott. ${ }^{1}$ GI Unit, Western General Hospital, Edinburgh, UK

Introduction The phenotype of elderly-onset IBD is poorly described and knowledge lags behind that of other age-groups. While not the dominant age-group in the disease population, those diagnosed over 60 will compose a larger proportion it as the general population ages over the next decade. The Montreal classification for IBD stratifies related to age into three categories, 40(A3) the study aims to ascertain if disease phenotype varies between those aged 40-59 and those $>60$ with possibility of an A4 group becoming viable if variance is noted.

Methods 1957 patients with IBD were identified using the IBD database at the Western General Hospital. We selected all those UC $(n=306)$ and CD $(n=135)$ diagnosed at age 40 and over (A3) and subdivided the group in to those over and under 60 . The diagnosis adhered to the criteria of Lennard-Jones and IBD was categorised according to the Montréal classification. Data collected included diagnosis, age at diagnosis, disease distribution, disease behaviour and smoking history. Follow-up were available for 5 years following diagnosis. Analysis of the groups was undertaken using $\chi^{2}$ and Fishers exact test.

Results Gender of CD patients in the different age groups (40-59; $\mathrm{M} / \mathrm{F}=53 / 96 . \quad>60 ; \mathrm{M} / \mathrm{F}=10 / 49 \mathrm{p}=0.0115)$ illustrated a higher proportion of women in the $>60$ group. CD patients who were diagnosed over the age of 60 had more isolated colonic disease at diagnosis. (L2; 40-59 N=28/77, >60 N=37/58, $\mathrm{p}=0.0032 \mathrm{~L} 3 ; 40-59$ $\mathrm{N}=15 / 77,>60 \mathrm{~N}=2 / 58 \mathrm{p}=0.0073)$. By 5 years of follow-up these differences were no longer significant. There was do difference in disease behaviour or smoking history. UC patients had more left sided disease and less distal disease at diagnosis (E1; 40-59 $\mathrm{N}=70$ / 204, $>60 \mathrm{~N}=20 / 102, p=0.0079 \mathrm{E} 2 ; 40-59 \mathrm{~N}=88 / 204,>60 \mathrm{~N}=57 /$ $102 \mathrm{p}=0.039)$. Smoking history showed a greater proportion of former smokers in the $>60$ group $(40-59 \mathrm{~N}=108 / 216,>60 \mathrm{~N}=70 /$ $107 \mathrm{p}=0.0058$ ).

Conclusion Disease phenotype at Dx in both UC and Crohn's differs in the over-60s at diagnosis but normalises to that of the $A 3$ population at follow-up. This data suggests that the introduction of an additional Montreal age classification, A4, would be clinically meaningful. Further analysis will demonstrate whether response to treatment differs in this age group.

Competing interests None declared.

\section{PM0-227 INFLIXIMAB INDUCTION THERAPY ALONE FOR ULCERATIVE COLITIS DOES NOT RESULT IN LONG TERM REMISSION}

doi:10.1136/gutjnl-2012-302514b.227

A J Brooks, ${ }^{*}$ K Robinson, A Wright, M E McAlindon, A Lobo. Gastroenterology, Sheffield Teaching Hospitals NHS Foundation Trust, Sheffield, UK

Introduction Infliximab (IFX) has demonstrated efficacy in moderate to severe ulcerative colitis (UC) with a reduction in short-term colectomy rates. ${ }^{1}$ In the UK, the National Institute for Health and Clinical Excellence (NICE) guidance relates to an induction course of three-doses for severely active ulcerative colitis. ${ }^{2}$ The aim of this study was to determine outcomes following IFX induction, including colectomy rate, use of corticosteroids (CS) or repeat IFX induction.

Methods Patients with UC at a single large teaching centre received IFX induction for UC requiring hospitalisation or when urgent consideration of surgery was given for resistant or rapidly relapsing disease were retrospectively reviewed (2008-2011). All patients had a Simple Colitis Activity Index (SCAI) at 0, 2, 6 weeks.

Results Twenty-seven patients were studied, median age 38 (range 23-64), with 17 (63\%) refractory to oral or intravenous CS (13 and 4 respectively). All received CS in the year preceding IFX; median 1 course (range 1-4). 23 (85\%) were on immunosuppression (IS) (16 thiopurines, 7 methotrexate), 3 intolerant or non-responsive and 1 naïve to IS. Twenty (74\%) received induction IFX alone. Median SCAI was 8 (range 4-13), 4 (range 0-9), 2 (range $0-10$ ) at 0,2 and 